\title{
Simulation of Effect of Pore Structure on Coke Strength Using 3-dimensional Discrete Element Method
}

\author{
Sun-Young KIM and Yasushi SASAKI \\ Graduate Institute of Ferrous Technology (GIFT), Pohang University of Science and Technology (POSTECH), 790-784, Hyo-ja \\ dong, Nam gu, Pohang, S. Korea.
}

(Received on January 29, 2010; accepted on March 26, 2010)

\begin{abstract}
The compression and cleavage simulations of cylindrical coke sample using 3-dimensional Discrete Element Method are carried out to investigate failure phenomena of coke, and the results are discussed by comparing with experimental results.

The following assumptions are applied to model coke. Coke matrix is an aggregation of primary particles which are connected by parallel bonds to be broken when the stress exceeds its corresponding bond strength. The voids between the primary particles are considered as pores and 'large pore balls' are inserted intentionally to investigate the effect of large pores on the coke strength by regulating their size and location.

According to the results, porosity is the most dominant factor for coke strength when it is compared with the strength of coke matrix texture. When large pores are distributed regularly they strengthen the coke compared with the randomly arranged cases. In the cleavage test, critical strength of coke sample is proportional to the exponential of porosity and minimum coke matrix area fraction of crack propagated cross sections.
\end{abstract}

KEY WORDS: coke strength; compression test; cleavage test; tensile test; porosity; pore; 3D DEM.

\section{Introduction}

The coke is strongly demanded to have high strength over compression or impact and not to make fines as much as possible to guarantee uniform gas and liquid flow. These days, the coke strength is measured and represented by Drum Index (DI) or Coke Strength after Reaction index (CSR), and actually these indexing methods are known to be well adapted to real industrial plant.

However these may not be applicable if the coke undergoes quite different producing condition from the conventional one. Also indexing methods are not proper to research for investigation of breakage mechanism or coke fines generation because they cannot explain the fundamental causes influencing the breakage mechanism. ${ }^{1,2)}$ For these reasons, many attempts have been made to measure the mechanical strength of processed coke sample directly through compression test, cleavage test (or it is called as tensile test) or bending test.

However, due to the non-uniform pore distribution, coke matrix texture and existence of several kinds of mineral, it is not easy to find out the regularity from the results of coke compression test or cleavage test, and continuum based simulation method is, therefore, not suitable for coke's failure simulation.

On the other hand, Discrete Element Method (DEM) regards coke particle as a lump of small primary particles which are strongly bonded to each other, and this feature makes it possible to reflect coke's irregularity on the coke's model. As a result, detailed modeling is possible and the simulation results are more real than other results using continuum based simulation tool.

In addition, DEM is well-developed and outstanding method to simulate dynamic movement of solid particles. To optimize iron making process, not only inside of blast furnace but also stocks charging steps including from conveyer belt to the top of blast furnace should be controlled. Currently DEM is adapted to iron-making process partially, but if the computational power is increased enough, processes can be connected in regular sequence, and it will be possible to see the whole iron-making process at a glance. In the case of this study, if breakage occurs at a single coke particle the pieces of broken coke also behave as independent particles and react with others, so it is possible to apply the breakage result to the afterward blast furnace simulation.

This study tries to measure the mechanical strength of cylindrical coke sample by compression and cleavage tests, and these experiments are simulated using 3 dimensional DEM. Also, the effect of large pores on the coke strength is examined by adjusting the number and location of large pores. 


\section{Simulation Setup}

\subsection{Coke Sample and Compression-equipment Mod- eling}

The coke sample using DEM consists of 1244-1955 small primary sphere-particles which have radius of $0.2-$ $0.4 \mathrm{~mm}$ following the Gaussian distribution. Each coke sample has cylindrical shape of $8 \mathrm{~mm}$ diameter and $8 \mathrm{~mm}$ height apparently. To generate the coke sample of desired shape, cylindrical mold is generated using walls, and then small primary particles fill the mold randomly. The coke model of this study is an aggregation of primary particles connected by parallel bond which is an elastic bridge to transmit both forces and moments between primary particles and also which is broken when normal or shear stress exceeds its corresponding strength. Although the coke model is an aggregation of rigid primary particles, the whole part of coke model cannot be considered as a rigid body due to the existence of parallel bond.

In order to describe the contact between primary particles, two models are applied; if two contacting particles are connected by parallel bond, parallel bond model is applied, and if two neighboring particles are free to move, linear model is applied to describe general contact between particles.

The sketchy concept of parallel bond is depicted at Fig. 1. 'Parallel bond'3) provides a finite-size piece of cementation-material connecting two primary particles. Parallel bond can be considered as a bunch of springs with constant normal and shear stiffnesses, uniformly distributed over a circular cross-section lying on the contact plane and centered at the contact point.

'Linear model'3) (see Fig. 2) controls constant stiffness and sliding behavior between 'primary particle and primary particle' or 'primary particle and wall' contact. For both parallel bond model and linear bond model, important properties are listed in Table 1. These values are determined by trials to show close simulation result with experimental result. More details are discussed in the Sec. 3.2.1. In this table, parallel bond radius factor $(\alpha)$ decides the ra-

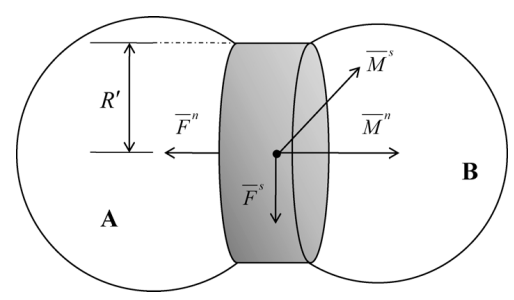

Fig. 1. Parallel bond described as a cylindrical cementation-material.

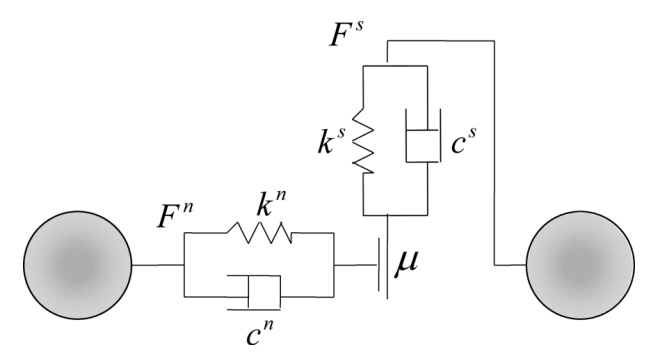

Fig. 2. Depiction of linear model of two neighboring particles. dius of parallel bond $\left(R^{\prime}\right)$ to be

$R^{\prime}=\alpha \times \min \left(R^{[\mathrm{A}]}, R^{[\mathrm{B}]}\right)$

where $R^{[\mathrm{A}]}$ and $R^{[\mathrm{B}]}$ are the radius of two contacting particles. Parallel bond stiffness is determined to give uniform stiffness with primary particle. As a result, parallel bond stiffness $\left(k^{\prime}\right)$ is decided to be

$$
k^{\prime}=\frac{0.5 \times k}{\overline{A^{\prime}}}
$$

where $k$ is primary particle stiffness and $\bar{A}^{\prime}$ is mean circular area of parallel bond, or, $\pi \times(0.3 \mathrm{~mm})^{2}$.

To investigate effect of pores on the coke strength, the following assumptions are used. First of all, the composition of coke sample is divided into two groups; coke matrix and pores. In other words, all the primary particles represent coke matrix of uniform property. Although real coke matrix contains several kinds of un-uniform texture, this assumption is unavoidable to increase the efficiency of modeling and to get the clear result of pores' effect.

Secondly, the void between the particles is considered as pore, and the porosity is defined as following formula.

$$
p=1-\frac{\sum \frac{4}{3} \pi r_{i}^{3}}{V_{\text {cyl }}}
$$

where $p$ is porosity, $r_{i}$ is the radius of each primary particle, and $V_{\text {cyl }}$ is volume of apparent cylinder. This formula does not include the volume of parallel bond because parallel bond is a virtual concept without visible form.

The pores are distinguished into two types. The first one is small voids between closely packed sphere particles and the second one is intentionally generated large voids which had been occupied by 'large pore balls'. In other words, some particles are generated temporary to substitute pores to make highly porous but strongly bonded material naturally. These particles are marked as black sphere in Fig. 3(a). When large pore balls are considered as occupying volume, the total volume of large pore balls is decided to make the coke sample's porosity be about $38 \%$ at every sample. For example, to make $60 \%$ porous sample, $38 \%$ of total cylinder's volume is occupied by small voids and the other $22 \%$ of total cylinder's volume is occupied by large pore balls (see Fig. 3). Because each of large pore balls has

Table 1. Micro-properties of primary particle and parallel bond.

\begin{tabular}{|c|c|}
\hline Number of primary particles & $1244 \sim 1955$ \\
\hline Primary particle radius ( $\left.\mathrm{R}^{\prime}\right)$ & $0.2 \sim 0.4(\mathrm{~mm})$ \\
\hline Primary particle density & $1500\left(\mathrm{~kg} / \mathrm{m}^{3}\right)$ \\
\hline Primary particle stiffness $(\mathrm{k})$ & $1.6(\mathrm{MPa})$ \\
\hline Friction coefficient & 1.0 \\
\hline Damping coefficient & 0.7 \\
\hline Parallel bond radius factor & 1.0 \\
\hline Parallel bond strength & $36\left(\mathrm{MN} / \mathrm{m}^{2}\right)$ \\
\hline Parallel bond stiffness $\left(\mathrm{k}^{\prime}\right)$ & $2.8 \mathrm{Tpa} / \mathrm{m}$ \\
\hline
\end{tabular}


same volume, if the number of large pores ball is chosen, their radius is decided automatically.

The compression equipment is expressed as two walls which are located at top and bottom of the coke sample. The compressive force is measured by these walls using built-in function. The walls move toward sample at strain rate, $2.0 \times 10^{-3} / \mathrm{s}$.

\subsection{Setup of Case Study}

\subsubsection{Compression Method}

To measure the coke strength, two kinds of test, the compression test and the cleavage test, are designed according to the compression direction (see Fig. 4). Compression test presses the coke sample at axial direction standing the sample. In this case, all the parts of coke sample get compressive stress evenly, so it is very easy to investigate the contribution of whole coke part on the strength. However, a main disadvantage is that it is not easy to predict and to observe where cracks would be occurring, especially at the 3-dimensional simulation.

Cleavage test lays down the coke sample and presses it across a diagonal direction. In this case, stress is concentrated at vertical cross section and tensile force is developed near the compressive axis. Because coke is a brittle material, when the first crack occurs it directly induces the other crack which propagates following the stressed area, so usually main crack propagation occurs along the vertical cross section. As a result, cleavage test narrows the portion of breakage occurring and makes it easy to observe the factors which influence the coke strength.

In the aspect of experimental conditions, compression test is easier method to get a general view about coke property rather than cleavage test, but tension which is devel-

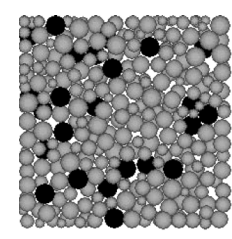

(a)

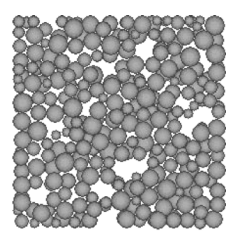

(b)
Fig. 3. Cross sections of coke sample with and without large pore balls. (a) $38 \%$ porosity with large pore balls, (b) desired porosity without large pore balls.

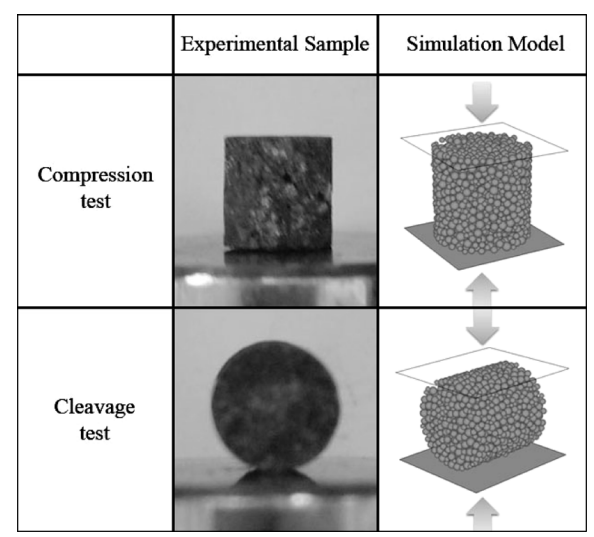

Fig. 4. Experimental sample and simulation model of compression test and cleavage test. oped during cleavage test is considered more dominant force factor in actual coke breakage process in blast furnace. Actually, when the same samples are tested, tensile strength is reported to be minimum $1 / 8$ of compression strength.

\subsubsection{Porosity Change and Arrangement of Large Pores}

For both compression test and cleavage test, coke sample's porosity changes from 45 to $60 \%$ by $5 \%$. As mentioned previously, $38 \%$ porosity is occupied by small voids between closely packed coke particles so that large pore balls volume is decided to be 7, 12, 17 and $22 \%$ of total volume of cylinder.

This study assumes that the stresses near the large pore would be most fatal for the breakage phenomena, so only large pore ball's number and locations are considered as influential factors. Therefore the coke samples are classified into two categories according to the distribution pattern of large pore balls; 'randomly distributed large pore balls sample' and 'arranged large pore balls sample' (these will be abbreviated to 'random sample' and 'arranged sample'). Arranged sample has large pore balls at 'body centered cubic' position maintaining same interval between large pore balls in both horizontal direction and vertical direction. According to the number of interval between large pore balls, samples of each porosity contain 9, 23, 43 and 113 large pore balls, but in some cases, the simulation is not carried out because as the space between large pore balls get closer or as the radius of large pore ball get bigger, it does not guarantee enough space for primary particles to settle and contact to each other. The exact positions of large pore balls in the arranged samples are marked in Fig. 5. The black circles show the positions at odd layer and gray circles show the positions at even layer from the outer layer.

Random samples also contain the same number of large pore balls to compare with arranged sample, but some cases also include 201 large pore balls to see the clear tendency of effect of the number of pores.

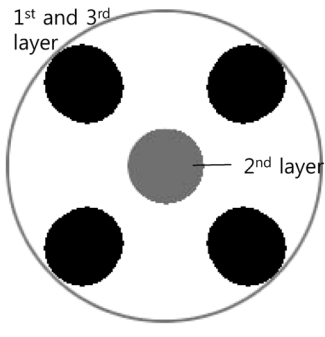

(a)

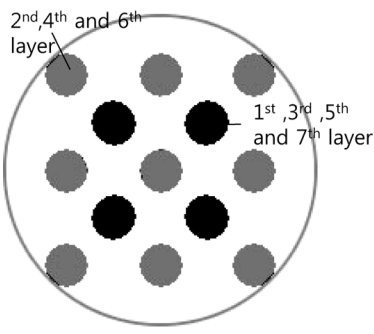

(c)

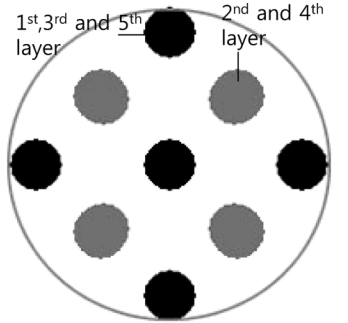

(b)

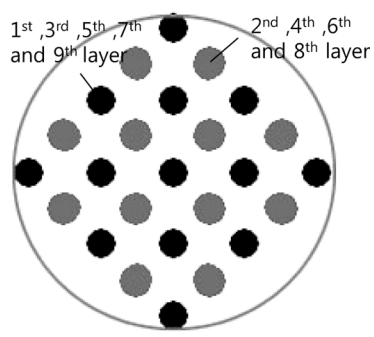

(d)
Fig. 5. Front view of arranged sample with 9, 23, 43 and 113 large pore balls. (a) 9 large pore balls, (b) 23 large pore balls, (c) 43 large pore balls, (d) 113 large pore balls. 
In addition, because the cleavage test splits the coke sample along vertical direction, the distribution pattern of large pore balls in the arranged sample also can be an influential factor to the test result. In order to investigate the effect of large pore balls which are located on or near the compressive axes, arranged samples are rotated $30^{\circ}$ or $45^{\circ}$ on the cylindrical axis and compared to non rotated sample.

\subsection{Experimental Setup}

As has been done by the simulation, actual compression test and cleavage test with real coke specimen were conducted to confirm the validity of simulation results. The size of sample was the same to that of simulation, or, cylindrical shape of $8 \mathrm{~mm}$ diameter and $8 \mathrm{~mm}$ height. The porosity was quite various from part to part but approximately 45-50\%. Among 8 samples, 5 samples were compressed at axial direction and the other 3 samples were compressed at diagonal direction. $100 \mathrm{kN}$ load cell was used to compress the specimens and strain rate was fixed at $2.0 \times 10^{-3} / \mathrm{s}$ for both cases. As both height and diameter are $8 \mathrm{~mm}$, the loading speed was $0.016 \mathrm{~mm} / \mathrm{s}$ for both compression and cleavage test.

\section{Results and Discussion}

\subsection{Experimental Result}

Figure 6(a) shows an example of stress-strain curves of

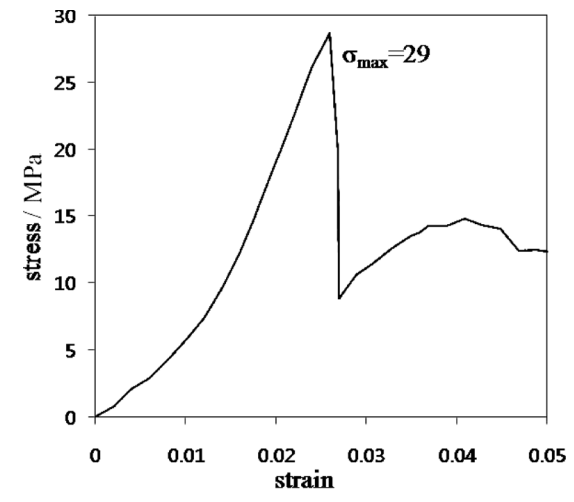

(a) compression experiment. The vertical axis is the compressive stress axis which is calculated by dividing compressive force by original circular contact area. Due to the heterogeneous texture of coke particles the experimental results quite fluctuated, but generally, peak compressive stress extends from 19.4 to $28.6 \mathrm{MPa}$ and breakage occurs at the strain range of $2.2-3.0 \%$.

Figure 7(a) is an example of stress-strain curves of cleavage experiment. In this case, the forced area is defined as vertical cross sectional area, diagonal $\times$ height, not a horizontal cross sectional area (even though the numerical value is same) or the half area of cylinder surface which is used at conventional coke tensile test. This disagreement is because it is very hard to define real contact area, and also the applied force acts complexly on coke sample. This paper regards the main cause of cleavage fracture is pulling force at vertical cross section towards side direction, and for this reason, adopt new forced area. If tensile stress values of this paper are converted according to the conventional area definition, they will have $64 \%$ value, but the graph shapes will not be changed.

As mentioned for compression experiment, the results shows fluctuation data, but generally, peak tensile force of cleavage test extends from 9.98 to $11.0 \mathrm{MPa}$ which is about $35-58 \%$ of that of compression test. Also the maximum peak stress is located at the strain range of $5.1-10.6 \%$.

While the stress-strain curve of compression test has

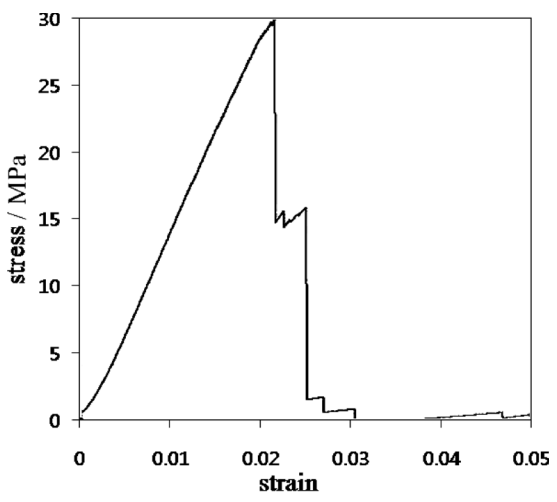

(b)

Fig. 6. Example of experimental and simulation results of compression test. (a) Experimental result, (b) simulation result.

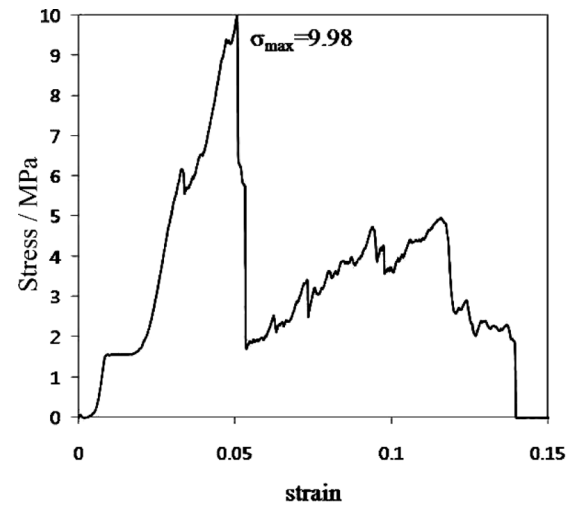

(a)

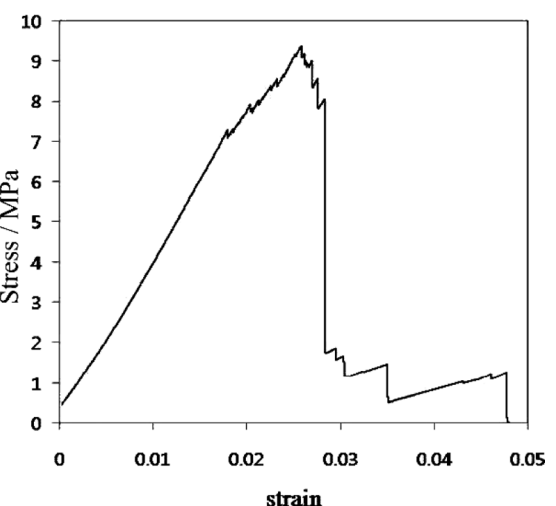

(b)

Fig. 7. Example of experimental and simulation results of cleavage test. (a) Experimental result, (b) simulation result. 


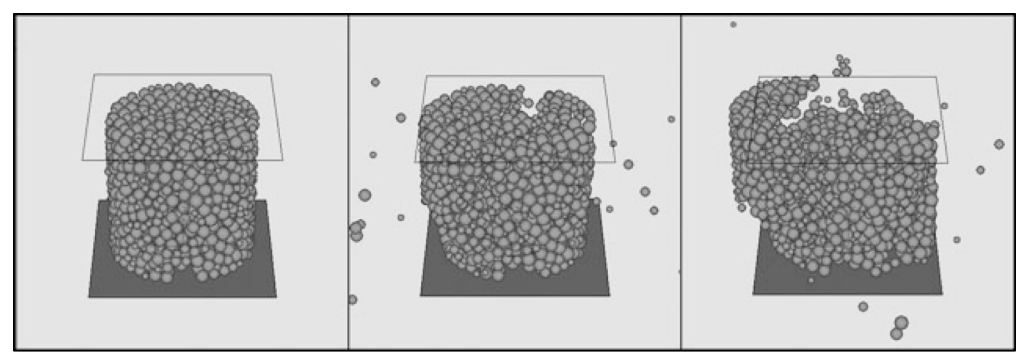

(a)

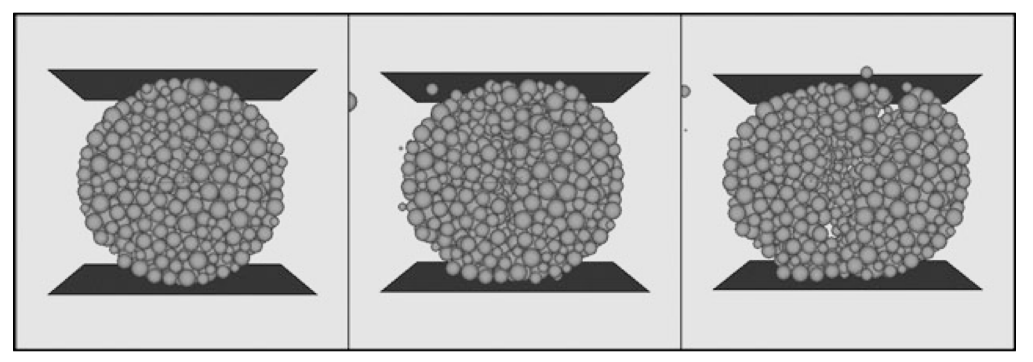

(b)

Fig. 8. Snapshot of simulation of breakage phenomena. (a) Compression test, (b) cleavage test.

quite smooth elastic region until it reaches to maximum peak and following sudden drop, that of cleavage test has bumpy slope until it reaches to maximum peak and following gradual drop.

Due to the excessive heterogeneity of coke, it is not possible to say that these several experimental data represent coke property sufficiently. These experiments are just executed to achieve the usual validity for the simulation result.

\subsection{Simulation Results and Discussion}

The simulation results are recorded as movie file and histogram of stress-strain curve. Figure 8 shows an example of snapshot of compression test and cleavage test. The simulation breakage pattern is very similar to that of experiment on the outside, but 3-dimensional simulation has some disadvantage that it is not easy to figures what happens at the inside of model. Therefore, the analysis of simulation is done by histogram of stress-strain curve.

\subsubsection{Compression Test}

Comparison with Experimental Result

Figure 6(b) shows an example of stress-strain curves of compression simulation. Since the experimental coke specimens have $45-50 \%$ porosity, a random sample of $45 \%$ porosity with 23 large pore balls is regulated to show slightly higher peak stress than that of experimental result, and peak stress occurs at around $2 \%$ strain range. As like the experimental result, the elastic region is quite clear, and sudden drop is shown after maximum peak. Based on this condition, the effect of porosity, the number of large pore balls and the distribution pattern of large pore balls are investigated relatively.

Effect of Porosity and the Number and Distribution Pattern of Large Pore B

The stress-strain curves of each porosity with 23 large pore balls are presented to show the effect of porosity on the coke strength in Fig. 9. This figure shows that increas-

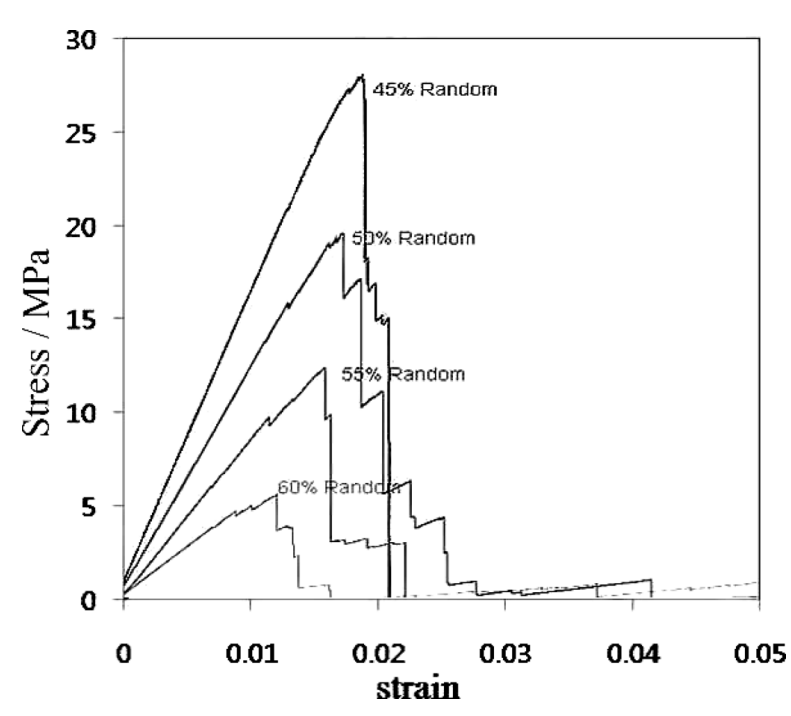

Fig. 9. Stress-strain curves of $45,50,55$ and $55 \%$ porosity sample with 23 large pores.

ing porosity reduces not only peak stress but also apparent stiffness gradually. That is because the decreased coke matrix volume means thinner coke matrix wall thickness. As a result, resistance of coke sample to the compressive force is reduced.

All peak stress and tendency lines of random sample and arranged sample are plotted in Figs. 10(a) and 10(b) according to the number of large pore balls. In this figure, R$45 \%$ means $45 \%$ porosity random sample and A-45\% means $45 \%$ porosity arranged sample. Obviously, the higher porosity, the lower peak stress of coke sample in both cases clearly.

In the aspect of distribution pattern of large pore balls, the arranged sample shows a little bit higher peak strength than that of the random sample at every porosity group. However in the aspect of the number of large pore balls, each porosity group shows no obvious increasing or de- 


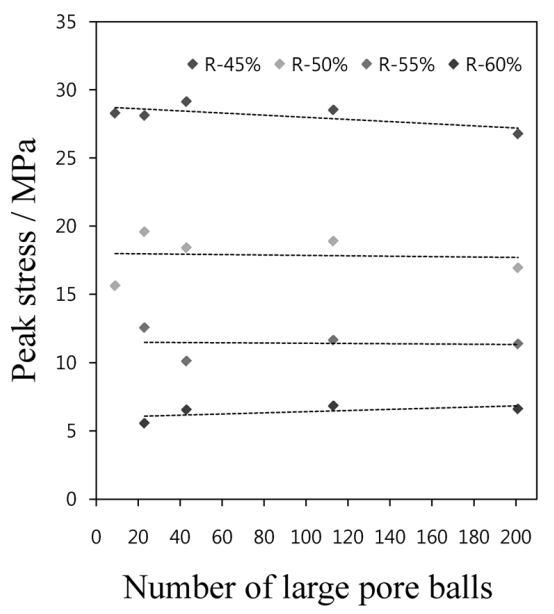

(a)

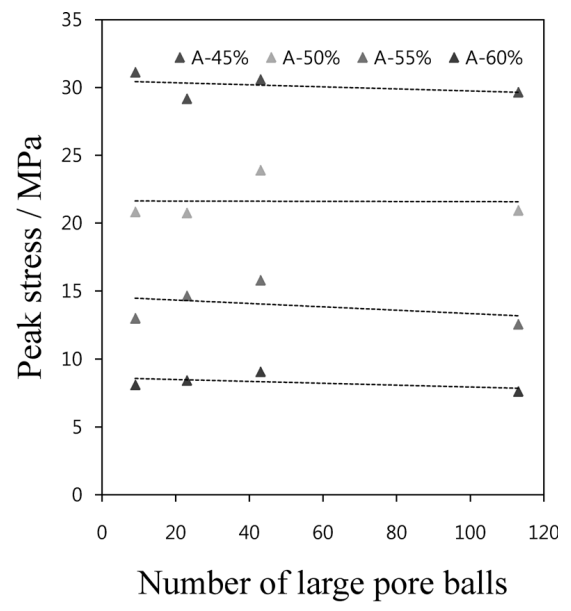

(b)

Fig. 10. Relation between peak compressive stress and number of large pore balls and porosity. (a) Peak compressive stress of random sample according to number of large pore balls and porosity. (b) Peak compressive stress of arranged sample according to number of large pore balls and porosity.

creasing tendency for each case although there are some deviations. Therefore, it is highly probable to conclude that the evenly distributed large pores can improve the strength of coke rather than the randomly distributed pores at fixed porosity, but the number, or size, of pore is not crucial factor to determine the coke strength.

As mentioned before, one of drawback of compression test is that it is not easy to predict where the breakage occurs and to find out the most influential factor to the breakage phenomena. Therefore more detailed discussion will be done at the next section with the result of cleavage test.

\subsubsection{Cleavage Test}

Comparison with the Results of Experiment and Compression Simulation

When 16 cleavage simulation results of random samples are compared with corresponding result of compression simulation, the peak stress of cleavage test result is reduced to $24-42 \%$ of peak stress of compression test result and main failure occurs at about $2.5-4.0 \%$ strain range (compare Figs. 7(a) and 7(b)).

This result shows that the simulated coke sample has a little bit smaller apparent stiffness to the diagonal directional compressive force than the actual specimen. This problem is due to relatively large primary particles and incorrect primary particle stiffness value. However due to the peak strength does not influenced by this error, using this sample to investigate the effect of pores onto the coke strength is possible.

Effect of Porosity and the Number and Distribution Pattern of Large Pore Balls

Cleavage test has been carried out as a name of 'tensile test' and the coke strength which is measured by cleavage test is called as 'tensile strength', because when a particle impacts to a wall or other particles, tensioned region is formed near the conical compressive stressed zone and the boundary which has the transition can have dominant effect for the crack generation. ${ }^{4)}$ For this reason, tensile strength has been investigated more often for a few decades and sev-

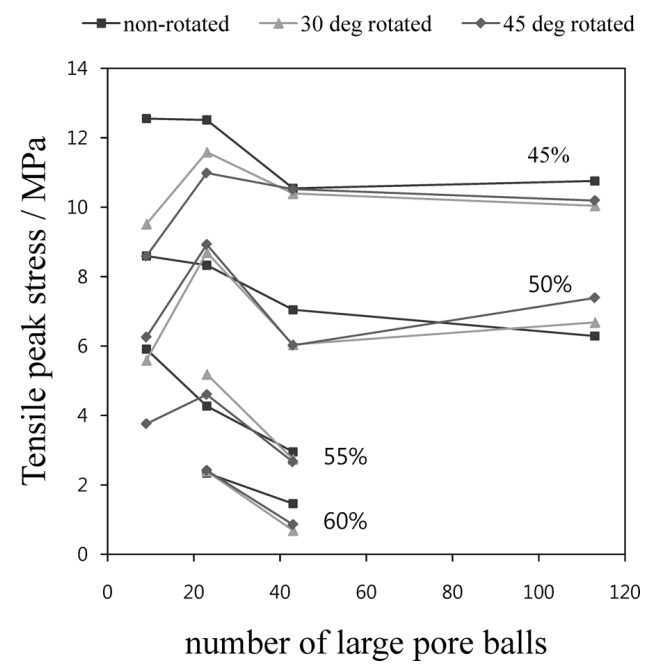

Fig. 11. The graph of tensile peak stress of arranged sample of each porosity according to the number of large pore balls.

eral possible equations have been suggested to build up the relation between coke tensile stress and porosity. Among them, Eq. (1) which was proposed by Knudsen ${ }^{5)}$ for the brittle polycrystalline specimens has been selected by many researchers and compared to others equations. ${ }^{6-9)}$

$$
\sigma=A \cdot \exp (-B \cdot p)
$$

where $\sigma$ is tensile stress (MPa), $p$ is porosity and $A$ and $B$ are positive constants.

This formula is also confirmed by using the simulation results of this study. The peak tensile stresses of non rotated, $30^{\circ}$ and $45^{\circ}$ counterclockwise rotated arranged samples are plotted at Fig. 11 according to the number of large pore balls. (Although the data are discontinuous, the plots are connected by lines to distinguish data of each group easily.) Above all, the peak tensile stress change due to porosity is outstanding. This is quite remarkable result because it can be explained that not only stressed cross section area but also whole part of coke sample contribute to 


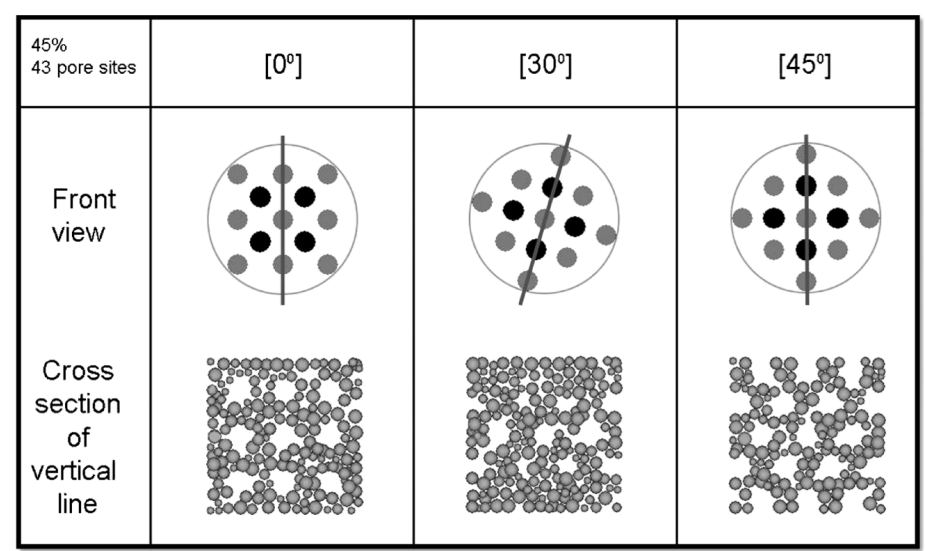

Fig. 12. Front view and vertical cross section of non rotated, $30^{\circ}$ and $45^{\circ}$ rotated arranged sample of $45 \%$ porosity with 43 large pore balls.

the breakage phenomena in cleavage test. It adds persuasive power to the view which is expressing the tensile stress as a function of porosity.

However, Eq. (1) does not include any other information such as wall thickness or pores number dispersion which can be powerful influential factors for the coke breakage, so a hypothesis of this paper is that tensile stress is closely related to the coke matrix area fraction of crack propagated cross section as well as porosity.

Figure 11 and Fig. 12 supply very important clue about the hypothesis. Figure 11 shows that the graphs of $30^{\circ}$ rotated sample and $45^{\circ}$ rotated sample are very similar for every cases but non-rotated sample has quite different graph pattern. As mentioned before, this study's DEM simulation result is quite fluctuating due to the simplified coke model, so the tendency is more important rather than absolute values to analyze data. If the fluctuation is considered, it is possible to say that $30^{\circ}$ rotated sample and $45^{\circ}$ rotated sample show almost same strength pattern. To investigate this phenomena more closely, the crack propagations are observed. Figure 12 shows front view and its vertical cross section of rotated arranged samples of $45 \%$ porosity with 43 large pore balls, and the lines on the front view pictures are the crack propagation direction, and these are similar for the other porosity cases. As the sample is compressed directly at the top and bottom of the sample, most of crack occurs at vertical direction, but if there is weaker path near the vertical cross section within low angle difference, the crack follows the inclined cross section. As a result, every crack of $30^{\circ}$ rotated sample occurs following the same cross section with $45^{\circ}$ rotated sample, but non rotated sample splits following the totally different cross section. This fact strongly supports that coke strength is closely related to the crack propagated cross section.

Actually until now several attempts were made to reveal the relationship between number of pores and strength through simulation with coke models which have well organized pores' distribution. For example, Ueoka el al. ${ }^{10)}$ investigated the effect of number of pores on the tensile fracture using two dimensional Rigid bodies-spring model (RBSM). In the paper, ${ }^{10)}$ the pores were arranged at the angular points of squares, so the samples had $4(2 \times 2)$, $16(4 \times 4)$ and $25(5 \times 5)$ pores, respectively, and porosity was fixed. According to the graph in the paper, the effect of

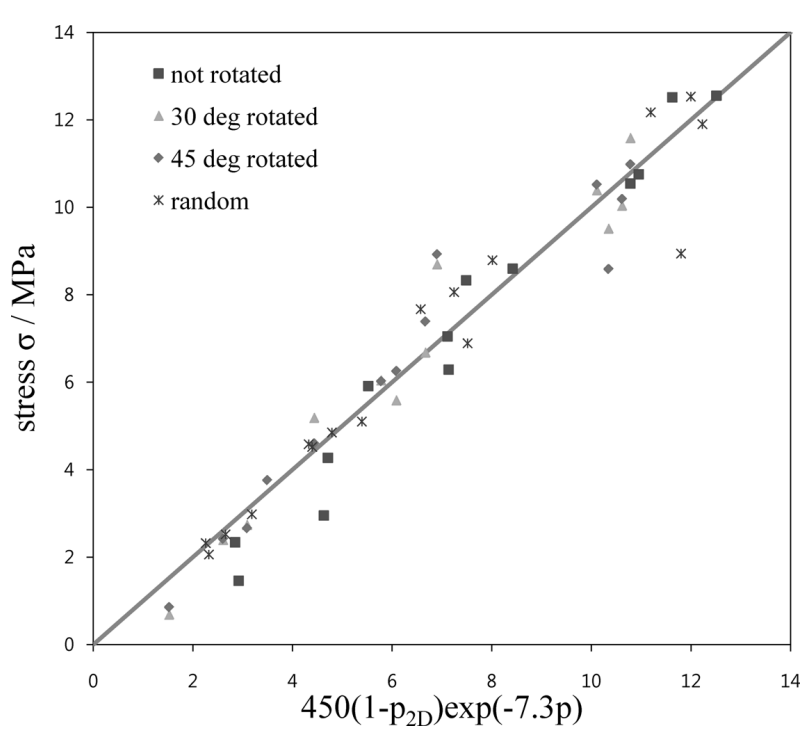

Fig. 13. The relation between tensile stress and $450\left(1-p_{2 \mathrm{D}}\right) \times$ $\exp (-7.3 p)$ of all simulation results.

number of pores on the strength is vague (although the author mentioned that the initial plastic deformation load is reduced for the small number of pores). However if the previous result is applied to this phenomena, it is unavoidable because the length of crack propagated cross line is the same for every case due to the square-type regular arrangement pattern of pores.

Therefore, the Eq. (1) is modified by inserting a term which is related to the area of coke matrix at the crack propagated cross section.

$$
\sigma=A \cdot\left(1-p_{2 \mathrm{D}}\right) \cdot \exp (-B \cdot p)
$$

where $p_{2 \mathrm{D}}$ is 2-dimensional porosity which could be estimated by the image of crack propagated cross section. Using all the simulation data of not only arranged samples but also random samples, Eq. (2) is tested in Fig. 13. In this figure, $A$ and $B$ values are decided to be 450 and 7.3 respectively for both arranged and random samples by trials. According to the figure, the Eq. (2) including the term of effect of coke matrix area fraction on crack propagated cross section is well fitted.

Actually according to the previous studies, $A$ is reported to be a characteristic value related to the coke matrix prop- 
Table 2. Comparison of coke strengthening effect between $10 \%$ decreased porosity sample and $10 \%$ increased coke-matrix bonding strength sample.

\begin{tabular}{|c|c|c|c|c|}
\hline \multicolumn{2}{|c|}{ Reference sample name } & variation & Coke-matrix & Porosity \\
\hline \multirow{4}{*}{$\begin{array}{c}\text { Porosity } \\
0.5\end{array}$} & Random 23 & \multirow[t]{4}{*}{$10 \%$} & $6.69 \% \uparrow$ & $43.6 \% \uparrow$ \\
\hline & Random 43 & & $0.38 \% \uparrow$ & $58.2 \% \uparrow$ \\
\hline & Random 113 & & $3.02 \% \uparrow$ & $51.0 \% \uparrow$ \\
\hline & Random 201 & & $3.96 \% \uparrow$ & $58.1 \% \uparrow$ \\
\hline \multirow{4}{*}{$\begin{array}{c}\text { Porosity } \\
0.55\end{array}$} & Random 23 & \multirow[t]{2}{*}{$10 \%$} & $0.398 \% \uparrow$ & $56.0 \% \uparrow$ \\
\hline & Random 43 & & $6.92 \% \uparrow$ & $81.9 \% \uparrow$ \\
\hline & Random 23 & \multirow[t]{2}{*}{$20 \%$} & $16.12 \% \uparrow$ & $123 \% \uparrow$ \\
\hline & Random 43 & & $22.1 \% \uparrow$ & $188 \% \uparrow$ \\
\hline
\end{tabular}

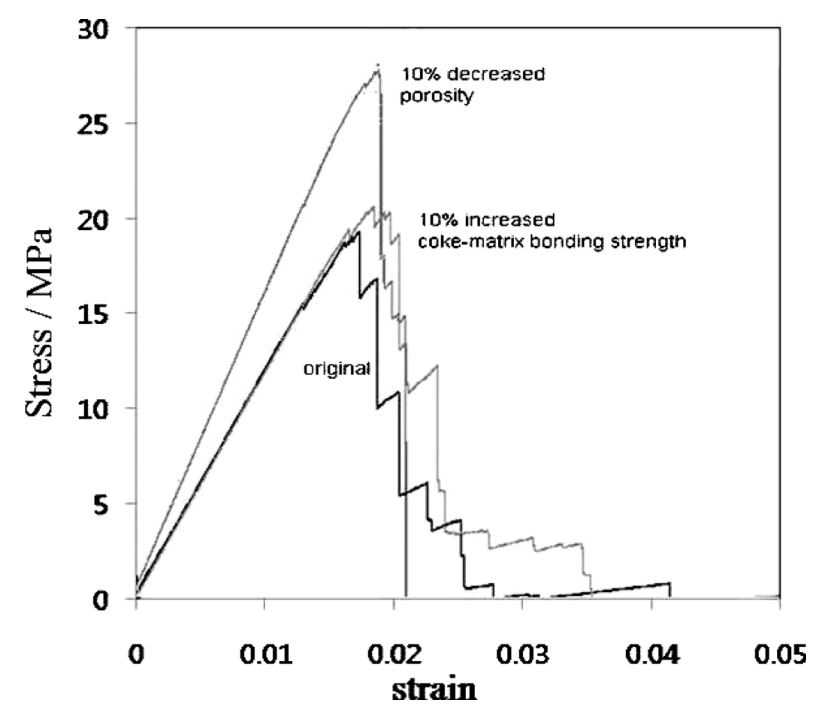

Fig. 14. Stress-strain curve comparison between $10 \%$ decreased porosity sample and $10 \%$ increased coke-matrix bonding strength sample based on the $50 \%$ porosity random sample with 23 large pores.

erty such as micro strength, ${ }^{7)}$ and $B$ is a pore shape factor. Especially in the paper of Patrick et $a l^{8)}$ the $B$ value is expressed as a factor representing the aspect ratio of influential pore. If the result is reflected, the random sample of the present paper should have bigger $B$ value than the arranged sample, but actually it is not.

As mentioned at experimental result section, this paper is adopting different forced area for tensile stress from the conventional one. If this study follows the previous definition for the tensile stress, the random sample shows surely bigger $B$ value than the arranged sample to reflect the aspect ratio of effective pores. However, as this paper is adopting different forced area, the effect of pore aspect ratio disappears, and only porosity and 2-dimensional porosity become influential factors for tensile stress. Further work is certainly required to find out the reason. by

For conclusion, the critical stress of coke sample is given

$$
\sigma_{\mathrm{c}}=A \cdot\left(1-p_{2 \mathrm{D}, \mathrm{Max}}\right) \cdot \exp (-B \cdot p)
$$

where $p_{2 \mathrm{D}, \mathrm{Max}}$ is maximum value among $p_{2 \mathrm{D}}$ of cross sections. The $p_{2 \mathrm{D}, \mathrm{Max}}$ value could be obtained statistically by image analysis or CT scan result.

\subsection{Comparison of Effects of Coke Matrix Bonding Strength and Porosity}

To improve the coke strength, two approaches are available: reducing porosity or increasing micro-strength of coke matrix. To find out which one would be more effective way, simple simulations are carried out. Figure 14 compares stress-strain curves of $10 \%$ decreased porosity and of $10 \%$ increased coke-matrix bonding strength based on the $50 \%$ porosity random sample with 23 large pore balls. According to the graph, the $10 \%$ increased bonding strength improves coke strength only by $6.7 \%$, whereas the $10 \%$ decreased porosity improves coke strength by $44 \%$. It is very intriguing result. Other cases also prove that decreasing porosity is much more effective method for coke strengthening compared with increasing coke matrix bonding strength. The results of other cases are listed in Table 2.

\section{Conclusion}

Coke is modeled using primary DEM sphere-particles and elastic parallel bonds. Although detailed expression is not possible, the simulation results of compression and cleavage (tensile) test show remarkable accordance with experimental results.

(1) When a coke sample has regularly distributed large pores, it shows a little bit higher strength rather than the coke sample with randomly distributed large pores for both compression and cleavage test.

(2) Tensile stress is the main factor to break coke particle rather than compressive stress, and the relation between tensile stress and coke property is

$$
\sigma_{\mathrm{c}}=450 \cdot\left(1-p_{2 \mathrm{D}, \mathrm{Max}}\right) \cdot \exp (-7.3 \cdot p)
$$

where $\sigma_{\mathrm{c}}$ is critical tensile stress, $p$ is porosity of coke, $p_{2 \mathrm{D}, \mathrm{Max}}$ is the maximum value among the 2-dimensional porosity which could be estimated by the image analysis or CT scan.

(3) To improve coke strength, decreasing porosity is much more effective method compared with increasing coke matrix bonding strength.

\section{REFERENCES}

1) M. Isobe, K. Suzuki, M. Tate and H. Kitagawa: Trans. Iron Steel Inst. Jpn., 21 (1981), 568.

2) H. Yamaoka, S. Suyama and K. Nakano: ISIJ Int., 43 (2003), 44. 
ISIJ International, Vol. 50 (2010), No. 6

3) Itasca Consulting Group Inc.: Theory and Background, PFC3D version 4.0, (2008).

4) M. Khanal, W. Schubert and J. Tomas: Granular Matter, 5 (2004), 177.

5) F. Knudsen: J. Am. Ceram. Soc., 42 (1959), 376

6) G. Pitt and J. Rumsey: J. Phys. D. Appl. Phys., 13 (1980), 969.

7) K. Nishioka and S. Yoshida: Trans. Iron Steel Inst. Jpn., 23 (1983),
387.

8) J. Patrick and A. Walker: Carbon, 27 (1989), 117.

9) M. Grant, A. Chaklader and J. Price: Fuel, 70 (1991), 181

10) K. Ueoka, T. Ogata, H. Hayashizaki, Y. Matsushita, H. Aoki, T. Miura, K. Fukuda and K. Matsudaira: Tetsu-to-Hagané, 95 (2009), 607. 